Service social

\title{
L'engagement des personnes ayant des incapacités à la formation en service social : pertinence, défis, et perspectives d'action
}

\author{
Élise Milot, Anne-Sophie Ruest-Paquette et Robert Labrecque
}

Volume 62, numéro 2, 2016

URI : https://id.erudit.org/iderudit/1038573ar

DOI : https://doi.org/10.7202/1038573ar

Aller au sommaire du numéro

Éditeur(s)

École de travail social et de criminologie de l’Université Laval

ISSN

1708-1734 (numérique)

Découvrir la revue

Citer cet article

Milot, É., Ruest-Paquette, A.-S. \& Labrecque, R. (2016). L'engagement des personnes ayant des incapacités à la formation en service social : pertinence, défis, et perspectives d'action. Service social, 62(2), 1-14.

https://doi.org/10.7202/1038573ar
Résumé de l'article

Au Québec, les travailleurs sociaux exercent souvent un rôle déterminant pour soutenir la participation sociale des personnes ayant des incapacités. Afin d'assurer le développement des compétences professionnelles nécessaires, solliciter l'engagement actif de ces personnes " expertes par expérience " apparaît comme une stratégie pédagogique riche de potentiel. Inspiré par les savoirs expérientiels et théoriques des coauteurs, cet article se veut une synthèse d'éléments appuyant la pertinence de miser sur cette stratégie dans la formation des futurs travailleurs sociaux. En guise d'entrée en matière, nous présentons une brève réflexion critique sur les termes employés dans les écrits pour référer à ces personnes, suivie d'une synthèse des principales retombées de cette stratégie pédagogique. Ensuite, différents aspects appuyant la pertinence de son utilisation dans des initiatives de formation s'inscrivant dans le champ du service social et du handicap ainsi que des recommandations en la matière sont proposées. L'écriture de cet article a été largement bonifiée grâce à une collaboration étroite avec une personne ayant des incapacités, impliquée dans la formation de futurs professionnels, aussi coauteure de ce texte. 


\title{
L'engagement des personnes ayant des incapacités à la formation en service social : pertinence, défis, et perspectives d'action
}

\author{
MILOT, Élise \\ Professeure adjointe \\ École de service social \\ Université Laval \\ RUEST-PAQUETTE, Anne-Sophie \\ Doctorante en service social \\ École de service social \\ Université Laval \\ LABRECQUE, Robert \\ Écrivain
}

\section{RÉSUMÉ}

Au Québec, les travailleurs sociaux exercent souvent un rôle déterminant pour soutenir la participation sociale des personnes ayant des incapacités. Afin d'assurer le développement des compétences professionnelles nécessaires, solliciter l'engagement actif de ces personnes « expertes par expérience » apparaît comme une stratégie pédagogique riche de potentiel. Inspiré par les savoirs expérientiels et théoriques des coauteurs, cet article se veut une synthèse d'éléments appuyant la pertinence de miser sur cette stratégie dans la formation des futurs travailleurs sociaux. En guise d'entrée en matière, nous présentons une brève réflexion critique sur les termes employés dans les écrits pour référer à ces personnes, suivie d'une synthèse des principales retombées de cette stratégie pédagogique. Ensuite, différents aspects appuyant la pertinence de son utilisation dans des initiatives de formation s'inscrivant dans le champ du service social et du handicap ainsi que des recommandations en la matière sont proposées. L'écriture de cet article a été largement bonifiée grâce à une collaboration étroite avec une personne ayant des incapacités, impliquée dans la formation de futurs professionnels, aussi coauteure de ce texte.

Mots-clés : engagement, expert par expérience, handicap, incapacités

\begin{abstract}
In the province of Québec, social workers play a determining role in supporting the social participation of people with disabilities. In order to ensure the development of necessary professional competencies, soliciting the active involvement of these " experts by experience » appears to be a pedagogical strategy with great potential, as argued in this article. First, we present a brief critical reflection on the terms used within the literature to refer to these people, which is followed by a synthesis of the main fallouts attributed to this pedagogical strategy. We then propose various aspects underlying the relevance of its use within training initiatives in the fields of social work and disabilities,
\end{abstract}


as well as recommendations in this matter. This article is in and of itself the fruit of a collaboration with a person living with disabilities who is involved in the training of future professionals. He is also a coauthor of this text.

Keywords: active involvement, expert by experience, handicap, disabilities 


\section{INTRODUCTION}

Le parcours de vie de tout être humain se caractérise par la présence de transitions, de défis et de projets. Pour les personnes ayant des incapacités, ces aspects importants de leur trajectoire sont bien souvent vécus avec l'apport de soins et services interpellant l'expertise de professionnels de différentes disciplines. À travers leurs interactions avec ces intervenants, les personnes ayant des incapacités développent une expertise à l'égard de leur condition et des pratiques les plus adéquates pour répondre à leurs besoins (Fox, 2008, 2011). Dès qu'elles sont en mesure de s'appuyer sur ces savoirs expérientiels pour informer autrui, influencer et évaluer la qualité des services offerts, elles deviennent de réels experts par l'expérience (Fox, 2011). Au cours des dernières années, plusieurs initiatives de formation ont fait appel à l'engagement actif (active involvement) de tels experts, ce que relève un bon nombre de recensions des écrits sur le sujet (Jha et al., 2009; Repper et Breeze, 2007; Robinson et Webber, 2013; Spencer et al., 2011; Towle et al., 2010). Or, parmi ces recensions, aucune ne s'intéresse exclusivement à des initiatives sollicitant l'engagement actif de personnes ayant des incapacités, hormis celle réalisée principalement par les deux premières coauteures de ce texte (Milot et al., soumis) ${ }^{1}$. Pourtant, le positionnement social de ces personnes, tout comme la nature et la diversité de leurs besoins, préoccupations et expériences, les distingue des autres groupes de personnes ayant recours à des soins et services. Les personnes ayant des incapacités semblent constituer des ressources pédagogiques hors pair qui peuvent non seulement faciliter le développement de compétences, mais aussi devenir garantes d'interventions centrées sur les forces et les capacités de chacun. Leur implication active dans la formation des futurs travailleurs sociaux ${ }^{2}$ doit être soutenue pour accélérer l'atteinte de résultats pertinents, en cohérence avec la mission et les valeurs de la profession. Cet article se veut une synthèse d'éléments appuyant la pertinence de cette stratégie pour la formation initiale en service social et des défis relatifs à son implantation. II s'inspire des savoirs théoriques des coauteurs, acquis à travers une recension exhaustive des écrits sur le sujet, et de leurs savoirs expérientiels, acquis à travers le codéveloppement d'un cours misant sur l'engagement actif de personnes ayant des incapacités (intellectuelles, motrices et sensorielles) à l'Université Laval (Milot, 2016). Avant de plonger dans le vif du sujet, l'éventail des termes employés pour désigner les personnes impliquées dans la formation sera d'abord dressé, notamment en vue de justifier nos choix terminologiques. Ensuite, il sera question de l'engagement actif en soi; nous définirons son but et ses retombées pour les apprenants, les instigateurs des initiatives pédagogiques et les personnes invitées et nous présenterons comment cet engagement peut se traduire pour une personne ayant des incapacités, aussi coauteure de ce texte. Puis, la pertinence de l'implication active des personnes ayant des incapacités dans la formation en service social sera explicitée. Finalement, nous effectuerons une synthèse de recommandations pour le développement d'initiatives s'inspirant de cette stratégie pédagogique.

\section{Terminologie}

Il est à noter qu'il n'existe aucun consensus dans les écrits scientifiques en ce qui a trait à la terminologie à privilégier pour désigner les personnes impliquées activement dans la formation des futurs professionnels, qu'elles aient ou non des incapacités. Les termes " patient », "client », « usager », « consommateur », « utilisateur » de services et « experts par l'expérience », sont tous

\footnotetext{
${ }^{1}$ L'article relatif à cette publication a été soumis à des fins de publication en juin 2016.

2 L'emploi du masculin dans ce texte a été choisi afin d'alléger la syntaxe.
} 
utilisés de manière interchangeable selon l'année de publication, la discipline concernée ou le pays où se situent les auteurs (Anghel et Ramon, 2009; McLaughlin, 2009; Spencer et al., 2011; Towle et al., 2010; Towle et Godolphin, 2013b). Chacun de ces termes réfère à une identité et à un positionnement distincts au sein d'une dynamique relationnelle comportant des enjeux de pouvoir (McLaughlin, 2009; Spencer et al., 2011; Towle et Godolphin, 2013b). En ce sens, la terminologie choisie n'est pas neutre. Au contraire, elle comprend une dimension à la fois personnelle et politique. Puisqu'ils traduisent des valeurs, des suppositions et des rapports chargés de sens, tant pour les personnes qu'ils désignent que pour celles qui les utilisent, les termes choisis sont susceptibles d'éveiller des sensibilités et de miner la communication entre ces deux parties (McLaughlin, 2009; Spencer et al., 2011; Towle et al., 2010; Towle et Godolphin, 2013b).

En service social, l'appellation « usagers de services » est souvent employée, et ce, surtout au Royaume-Uni. Selon McLaughlin (2009), ce terme réduit la personne à un statut passif de bénéficiaire de services et neutralise la diversité et la complexité des problématiques et des individus qu'il rassemble, tout en excluant ceux qui, pour une raison ou une autre, sont incapables d'accéder aux services ou choisissent de ne pas y recourir.

En ce qui a trait à l'expression « expert par expérience », celle-ci est parfois jugée trop inclusive. En effet, son utilisation peut référer à toute personne ayant eu recours à des services, adaptés ou non à ses besoins. Ce terme pourrait aussi qualifier tout individu qui n'a pas reçu de services en dépit d'une demande, celui qui choisit de ne pas y recourir ou encore, tout proche impliqué dans la vie d'une personne qui reçoit des services (McLaughlin, 2009). Cela dit, comment différencier l'individu qui est expert par expérience de celui qui ne l'est pas? Sur la base de quels critères établit-on cette distinction? Qui détient l'autorité permettant d'établir ces critères et d'émettre un jugement en ce sens ? D'après Fox (2008), ce sont les capacités réflexives, les connaissances, la confiance, l'autonomie et l'autodétermination de la personne qui assurent la médiation entre ses expériences et son expertise. C'est ainsi que des expériences individuelles sont traduites sous la forme d'une expertise plus vaste.

À la demande des personnes impliquées dans leur étude, Anghel et Ramon (2009) ont plutôt adopté le terme «consultant ». Toutefois, ce mot, qui réfère à un rôle spécifique dans le contexte d'une initiative précise, ne permet pas d'illustrer la dynamique bidirectionnelle entre les personnes invitées et les instigateurs de l'initiative en question.

Pour l'heure, il semble n'exister aucun article scientifique en français portant sur l'engagement actif à la formation dans les écrits sur le sujet. II n'y a donc pas de précédent lexical francophone en la matière. Comme le conseille McLaughlin (2009), l'idéal demeure, bien sûr, de céder la parole aux personnes concernées afin qu'elles décident elles-mêmes comment elles souhaitent être nommées et décrites. Or, dans le cadre de cet article, nous avons choisi les appellations «personnes ayant vécu des expériences significatives en matière de soins et services » (plus générale) et «personnes ayant des incapacités " (plus spécifique). Lorsque nous cherchons à insister sur l'expertise de ces personnes à l'égard de leur condition et des pratiques les plus adéquates pour répondre à leurs besoins, nous utilisons le terme « experts par expérience». 


\section{L'engagement actif}

L'engagement actif de personnes ayant vécu des expériences significatives en matière de soins et services s'appuie sur la reconnaissance de l'unicité de leur expertise et sur le bien-fondé de son intégration au sein de la formation des professionnels futurs ou en exercice (Beresford, 2000; Fox, 2011; Jha et al., 2009; Spencer et al., 2011; Towle et al., 2010). Cette approche pédagogique, dont la popularité ne fait que croître depuis le début des années 2000 , est utilisée principalement en sciences de la santé, mais aussi en sciences humaines et sociales, et ce, surtout au Royaume-Uni où des politiques le prescrivent (Duffy et Hayes, 2012; Jha et al., 2009; Repper et Breeze, 2007; Robinson et Webber, 2013; Spencer et al., 2011; Towle et al., 2010; Towle et Godolphin, 2013a, 2013b). Bien qu'elle soit davantage intégrée au sein des programmes universitaires de premier cycle, elle est tout de même utilisée à travers tout le continuum de formation professionnelle (études techniques, cycles supérieurs universitaires et formation continue au sein des établissements) (Spencer et al., 2011; Towle et Godolphin, 2013b).

Avant d'examiner la pertinence de l'engagement actif des personnes ayant des incapacités dans le cadre d'initiatives de formation en service social, nous allons d'abord examiner en quoi consiste l'engagement actif de personnes ayant vécu des expériences significatives en matière de soins et services (ayant ou non des incapacités) et en survolerons les retombées en s'intéressant au point de vue des apprenants, des instigateurs des initiatives et des personnes impliquées.

\subsection{Qu'est-ce que l'engagement actif dans l'enseignement?}

L'engagement actif décrit l'implication de personnes possédant une expertise acquise à travers leur expérience de la maladie, d'iniquités ou de limitations dans l'enseignement, le développement de curriculums et l'évaluation des apprenants. Les personnes concernées sont conscientes du rôle et des responsabilités éducatives qui leur sont assignés (Baldwin et Sadd, 2006; Repper et Breeze, 2007; Robinson et Webber, 2013; Spencer et al., 2011; Towle et al., 2010; Towle et Godolphin, 2013b). L'engagement actif peut aussi référer à toute démarche de consultation, de planification de cours, de production de matériel didactique, de gestion de programme, de recrutement et de sélection des apprenants participant à une initiative de formation en particulier. Sous sa forme la plus accomplie, cet engagement s'actualise à travers un partenariat équitable entre les personnes sollicitées et les instigateurs des initiatives en question dans toute prise de décision concernant les contenus et la mise en œuvre d'une démarche pédagogique collaborative (Spencer et al., 2011; Towle et al., 2010). Cependant, un tel partenariat peut se buter à certains freins en milieu institutionnel, tels que : (1) une réglementation et un système d'attribution du financement inflexibles empêchant la mise en place d'adaptations requises pour assurer l'implication maximale des personnes invitées; (2) la rigidité des cursus de cours; (3) la survalorisation de l'expertise théorique au sein de la culture institutionnelle, comparativement à l'expertise expérientielle; (4) la promotion persistante de la professionnalisation des personnes invitées; (5) l'obligation, pour les personnes impliquées, de participer à un processus de recrutement et de sélection, voire de formation - lequel peut s'avérer plus ou moins flexible et adapté (Baldwin et Sadd, 2006; Repper et Breeze, 2007; Towle et Godolphin, 2013a). Par conséquent, plutôt que de miser sur la notion de partenariat équitable entre les personnes impliquées et les instigateurs des initiatives, viser la création d'une relation misant sur la complémentarité des expertises paraît plus réaliste (Fox, 2011). Dans cette optique, la notion d'alliance, référant à une collaboration plutôt qu'à une relation égalitaire, est préférable dans la mesure où elle renvoie à une 
relation significative fondée sur le respect et la valorisation de la contribution de chacun, en dépit d'une distribution inégale du pouvoir et des responsabilités (Baldwin et Sadd, 2006).

L'engagement actif des personnes ayant des incapacités à la formation des futurs professionnels peut prendre différentes formes. À titre d'exemple, les personnes peuvent être invitées à interagir avec des petits groupes d'étudiants issus de différents programmes d'études lors d'un atelier participatif ou d'un programme de mentorat (Jha et al., 2009; Repper et Breeze, 2007; Robinson et Webber, 2013; Towle et al., 2010).

\subsection{Quelles retombées?}

Jusqu'à présent, aucune étude longitudinale n'a été réalisée pour mesurer les retombées à long terme de l'engagement actif des personnes ayant vécu des expériences significatives en matière de soins et services dans les initiatives de formation; seules les retombées à court terme sont connues pour le moment (Jha et al., 2009; Spencer et al., 2011; Towle et al., 2010). Une synthèse des principales retombées chez les apprenants, les instigateurs des initiatives et les personnes impliquées est présentée aux sections suivantes.

\subsubsection{Chez les apprenants}

Les apprenants sont généralement satisfaits des initiatives faisant appel à l'engagement actif des personnes ayant vécu des expériences significatives en matière de soins et services (Anghel et Ramon, 2009; Jha et al., 2009; Repper et Breeze, 2007; Spencer et al., 2011; Towle et al., 2010; Towle et Godolphin, 2013b). En plus d'apprendre dans le contexte d'une démarche pédagogique authentique et significative axée sur des expériences vécues, telles que relatées d'un point de vue endogène (de l'intérieur), les apprenants en ressortent avec un sentiment d'efficacité professionnelle accrue (p. ex. : augmentation du sentiment de confiance et diminution de l'anxiété, conscience de leur rôle et de l'impact de leurs interventions); deviennent plus sensibles aux besoins de populations en situation de vulnérabilité, ce qui influe positivement sur leurs attitudes (p. ex. : intégration des valeurs et croyances professionnelles, dont l'empathie); acquièrent des connaissances spécifiques ( $p$. ex. : au sujet d'un diagnostic en particulier) et développent des compétences nécessaires à l'exercice de leur profession ( $p$. ex. : approche centrée sur la personne; habiletés communicationnelles - incluant l'écoute active et la capacité d'adapter son niveau de langage; facilité accrue à faire des liens entre la théorie et la pratique; etc.) (Anghel et Ramon, 2009; Baldwin et Sadd, 2006; Duffy et Hayes, 2012; Jha et al., 2009; Repper et Breeze, 2007; Robinson et Webber, 2013; Spencer et al., 2011; Towle et al., 2010).

Towle et ses collaborateurs (2010) identifient des retombées d'un autre ordre. Ils constatent d'abord la présence de difficultés associées à la gestion du temps lors de projets collaboratifs. Ils relèvent aussi le sentiment d'être un fardeau pour les personnes impliquées et la difficulté à gérer leur sentiment d'attachement, ce qui est d'autant plus affligeant lorsque la santé des personnes se détériore. Aussi, comme cet engagement actif engendre des remises en question quant aux pratiques à privilégier, aux valeurs personnelles et professionnelles, aux compétences à développer et à la distribution du pouvoir, l'expérience peut s'avérer troublante pour certains apprenants, lesquels peuvent se montrer réfractaires à la démarche proposée (Baldwin et Sadd, 2006). 


\subsubsection{Chez les instigateurs des initiatives}

Les instigateurs des initiatives, qui assurent davantage le rôle de facilitateur, sont satisfaits d'avoir utilisé cette stratégie pédagogique auprès des apprenants qui leur paraît fort efficace pour assurer le développement de connaissances, d'attitudes et de compétences pouvant être réinvesties dans la pratique professionnelle (Anghel et Ramon, 2009; Towle et al., 2010). Toutefois, certains s'inquiètent de ses effets dans la vie des personnes invitées (Repper et Breeze, 2007) et de l'ampleur de la charge de travail et du temps à investir pour reconduire ou développer d'autres initiatives de formation s'inspirant de cette stratégie pédagogique (Spencer et al., 2011).

\subsubsection{Chez les personnes impliquées}

De manière générale, les personnes invitées souhaitent que leur expertise soit mise à contribution et sont satisfaites de leur implication : elles apprécient notamment redonner à la communauté en partageant leurs expériences, tout en faisant ressortir les enjeux qu'elles considèrent prioritaires. De plus, elles apprécient l'ouverture d'esprit et l'engagement des apprenants à la démarche collaborative, et reconnaissent l'apport de leur expertise au développement des compétences des futurs professionnels (Anghel et Ramon, 2009; Baldwin et Sadd, 2006; Duffy et Hayes, 2012; Repper et Breeze, 2007; Spencer et al., 2011; Towle et al., 2010). En outre, leur engagement actif à la formation génère des répercussions positives sur leur estime de soi et leur appropriation de leur propre pouvoir (Baldwin et Sadd, 2006; Fox, 2011; Jha et al., 2009; Repper et Breeze, 2007; Robinson et Webber, 2013; Spencer et al., 2011).

Cela étant dit, le récit récurrent de leur histoire de vie (ou le dévoilement de leur vie privée), la crainte d'être jugés, la manière dont leur récit est représenté dans les travaux des apprenants, l'utilisation du jargon professionnel, l'incompréhension d'autrui et la peur de décevoir sont des facteurs susceptibles d'accroître leur niveau de stress et d'anxiété (Fox, 2011; Jha et al., 2009; Repper et Breeze, 2007; Spencer et al., 2011; Towle et al., 2010). Baldwin et Sadd (2006), qui constatent que les personnes invitées effectuent parfois des évaluations contradictoires à l'égard de leur implication, rapportent aussi que celles-ci se sentent parfois diminuées, prises au dépourvu ou confuses.

Voici un bref résumé portant sur l'expérience d'engagement à la formation des étudiants de monsieur Robert Labrecque, coauteur de ce texte.

\section{Engagement actif d'un expert par expérience}

Je m'appelle Robert Labrecque. Je suis un écrivain âgé de 59 ans, grand-père et fier de l'être, qui demeure avec sa conjointe dans une coquette demeure de Québec. Atteint d'une maladie neuromusculaire dégénérative, je me déplace en fauteuil motorisé depuis l'âge de 20 ans. Aussi, je milite au sein de différentes associations de défense des droits depuis plus de trente-huit ans.

Pour moi, demeurer à domicile, ainsi que gérer et choisir mes services, est d'une très grande importance. Dans ce but, j'embauche moi-même des étudiants issus de différentes disciplines ( $p$. ex. : travail social, inhalothérapie, sciences infirmières) pour recevoir mes services à mon domicile grâce au programme d'allocation directe du chèque emploi service du ministère de la Santé et des Services sociaux. Parmi les 
différentes responsabilités que cette gestion suppose, celle que je préfère est celle d'assurer la formation des étudiants qui m'offrent leur assistance à mon domicile. Bien que mes "enseignements » visent d'abord le développement d'habiletés liées à des tâches techniques (p.ex. : faire un transfert pour me déplacer du fauteuil au lit; manier l'équipement technique), ils me permettent aussi de faciliter l'acquisition de précieux savoir-agir chez ces étudiants. Au fil de nos rencontres, je les amène graduellement à prendre le temps d'écouter mes besoins, ce qui m'apparaît fondamental puisque je suis le réel expert de ma condition. Nous développons, petit à petit, une réelle alliance caractérisée par une grande ouverture, de la complicité, une bonne dose d'humour et un respect sans limites de mes droits et capacités.

Depuis janvier 2015, je mets mes savoirs expérientiels à profit dans le cadre d'une démarche participative visant la création d'un cours à l'Université Laval, ce qui me remplit d'enthousiasme. Jusqu'à présent, j'ai été sollicité dans la création de capsules vidéo portant sur ma vision de la participation sociale et sur mon implication dans les associations de défense des droits des personnes handicapées des années 1970 jusqu'à aujourd'hui. J'ai aussi partagé mes idées et mes points de vue lors de six ateliers au sein d'un comité consultatif de personnes ayant différents types d'incapacités. Au printemps 2016, j'ai participé au cours «Collaboration interprofessionnelle et soutien à la participation sociale dans le champ du handicap ». J'ai animé des périodes de discussion au sein de petites équipes d'étudiants, ce qui les a grandement aidés dans l'élaboration de leurs travaux. Je suis bien heureux d'avoir participé à ce projet, car j'ai le sentiment d'avoir contribué à la formation de professionnels réflexifs, créatifs, empathiques et humains.

\section{Pertinence pour la formation des futurs travailleurs sociaux}

Afin d'être estimé pertinent, l'engagement actif de personnes ayant vécu des expériences significatives en matière de soins et services doit être substantiel, notamment en service social (Robinson et Webber, 2013). De manière générale, les raisons évoquées pour justifier un tel engagement sont les suivantes: (1) les politiques gouvernementales et professionnelles l'encouragent ou l'exigent (selon le contexte); (2) les experts le recommandent dans les écrits scientifiques; (3) les universités détiennent une responsabilité sociale à l'égard des personnes et des populations en situation de vulnérabilité; (4) l'expertise de ces personnes (savoirs expérientiels) est complémentaire à celle des instigateurs des initiatives (savoirs théoriques), ce qui enrichit les curriculums de formation; (5) les communautés véhiculent un fort désir de s'impliquer dans la formation des professionnels (futurs ou actifs) afin d'y faire valoir leurs attentes et préoccupations; (6) l'engagement actif des personnes sollicitées rehausse la pratique professionnelle (Baldwin et Sadd, 2006; Jha et al., 2009; Repper et Breeze, 2007; Robinson et Webber, 2013; Spencer et al., 2011; Towle et al., 2010; Towle et Godolphin, 2013b). Étant donné leur bagage d'expériences et de connaissances variées, plusieurs experts par expérience sont particulièrement motivés à s'impliquer dans la formation de futurs professionnels, en partageant des exemples concrets d'interventions vécues, des conseils pratiques et des rétroactions pertinentes (Jha et al., 2009). Quelle que soit la justification, cet engagement actif fait la promotion d'une approche centrée sur la personne, qui met à l'avant-plan ses préférences, ses 
préoccupations, ses expériences et ses valeurs au cœur du processus décisionnel, eu égard aux soins et services permettant de répondre à ses besoins (Jha et al., 2009; Spencer et al., 2011; Towle et al., 2010; Towle et Godolphin, 2013b). Ainsi, l'engagement actif incite à conceptualiser autrement la notion d'expertise et remet en cause la distribution hiérarchique du pouvoir entre les instigateurs des initiatives, les apprenants et les personnes ayant vécu des expériences significatives en matière de soins et services (Fox, 2011; Towle et Godolphin, 2013a). Plutôt que d'être réduites à des objets au sujet desquels on apprend, ces personnes y sont perçues comme des acteurs avec lesquels on apprend. En d'autres termes, les apprentissages se réalisent grâce à l'alliance entre toutes les parties impliquées et grâce aux interactions qu'elle permet (Bleakley et Bligh, 2008). En effet, l'implication directe des personnes expertes par expérience engendre nécessairement des changements à l'égard de la relation de pouvoir, des rôles et du sens attribué à la relation entre les futurs professionnels et les personnes qui reçoivent des soins et services, si bien que chacun a la possibilité de s'exprimer dans le respect de son authenticité et de son autonomie (Anghel et Ramon, 2009; Bleakley et Bligh, 2008). Ce faisant, les capacités et le potentiel des personnes impliquées sont mis de l'avant et leur pouvoir d'agir est valorisé (Anghel et Ramon, 2009). Du coup, les apprenants ont l'occasion d'apprendre en fonction des priorités établies par ces personnes qui préconisent le développement de pratiques professionnelles humanistes (Repper et Breeze, 2007). Un tel renversement de pouvoir est nécessaire pour accéder à une meilleure compréhension et prise en compte de la perspective des personnes ayant recours à des soins et services, ce qui paraît d'autant plus important en service social.

Reconnaissant le rapport d'interdépendance entre la personne et son environnement social, la mission première des travailleurs sociaux est de promouvoir et de faciliter le bien-être des personnes, groupes et collectivités en état de vulnérabilité, c'est-à-dire en situation de marginalisation, d'exclusion ou d'oppression (Colby et Dziegielewski, 2010; Bouquet, 2004; Brodeur et Berteau, 2008; Van de Sande et al., 2002). Le service social soutient des idéaux humanistes et démocratiques (Bouquet, 2004; Brodeur et Berteau, 2008) et ses valeurs centrales sont le « respect de l'égalité, de la valeur et de la dignité de tous » (Fédération internationale des travailleurs sociaux, 2000, citée dans Van de Sande et al., 2002, p. 11). La tolérance, l'autodétermination, la compassion, la responsabilité et l'empathie figurent également parmi ses valeurs dominantes (Bouquet, 2004). L'exclusion des personnes ayant vécu des expériences significatives en matière de soins et services des milieux qui sont imputables de leur qualité (p.ex. : universités, établissements publics) paraît alors incompatible avec les valeurs du service social (Beresford, 2000). Ces milieux doivent conséquemment non seulement promouvoir ces valeurs, mais aussi les modeler; l'engagement actif des personnes dans des initiatives de formation peut s'inscrire dans un tel effort de modelage. À ce sujet, cet engagement est de plus en plus répandu dans les initiatives de formation en service social (Duffy et Hayes, 2012). Le désir de tendre vers des alliances authentiques entre personnes invitées et futurs travailleurs sociaux est enraciné dans la promotion d'une perspective inclusive et égalitaire. II témoigne également d'une ferme volonté de faciliter l'implication de ces personnes dans une démarche d'amélioration continue des services offerts et d'un engagement à soutenir leur participation aux changements sociaux auxquels aspire la profession (Anghel et Ramon, 2009). De telles alliances peuvent aussi influer sur la théorisation au sein de la discipline.

Rappelons que la théorisation en service social doit notamment s'appuyer sur les connaissances et expériences de personnes ayant vécu des expériences significatives en matière de soins et services, incluant celles qui vivent avec des incapacités. Comme le souligne Beresford (2000), il paraît donc 
primordial d'aménager des espaces discursifs institutionnels avec elles, tout en valorisant leur participation à des plateformes, qu'elles soient écrites ou orales, formelles ou informelles, individuelles ou collectives. La formation de futurs professionnels constitue l'une de ces plateformes discursives. En effet, l'engagement actif de personnes ayant des incapacités dans ce contexte leur permet justement de faire valoir leurs connaissances et expériences. Tandis que la théorisation en service social a pour objet premier l'avancement intellectuel au profit de la discipline (p. ex. : pratiques, politiques, enseignement et recherche), la théorisation des mouvements de personnes ayant vécu des expériences significatives en matière de soins et services est davantage associée à l'action et au changement social (Beresford, 2000), qui sont aussi compatibles avec la visée globale du service social. Par conséquent, en plus de pallier les limites relatives aux savoirs académiques en matière d'incapacités dans les programmes de formation et de favoriser l'optimisation des pratiques centrées sur les besoins de ces personnes, le recours à leur perspective dans l'enseignement est également propice au développement de théories pouvant engendrer des transformations sociales significatives (Beresford, 2000).

De surcroît, la dimension collaborative des initiatives de formation fondées sur un tel engagement permet l'instauration d'un dialogue entre les personnes impliquées, les apprenants et les professionnels. D'après Brodeur et Berteau (2008), le dialogue favorise le respect de la dignité humaine et de l'autodétermination (deux valeurs dominantes en service social), facilite la résolution de conflits et maximise la coopération entre interlocuteurs. II consiste plus exactement en un « échange authentique, réciproque et égalitaire » tributaire de trois conditions fondamentales, soit: (1) «la reconnaissance et l'acceptation des principaux acteurs en tant qu'interlocuteurs légitimes »; (2) «la reconnaissance et l'acceptation de la différence » et (3) «la capacité de reconnaître que l'autre a la même valeur [que soi] sur le plan humain et qu'il est lui aussi capable de dialogue » (Malherbe, 1990, dans Brodeur et Berteau, 2008, p. 258-259). Cela dit, nous avançons donc que l'engagement actif des personnes ayant des incapacités dans des initiatives de formation en service social est une stratégie pédagogique pouvant permettre l'émergence d'un dialogue authentique, réciproque et égalitaire, dont la pratique permet aux étudiants d'être mieux outillés pour poursuivre ce dialogue dans le cadre de leurs interventions professionnelles.

La section qui suit présente quelques recommandations pour le développement de telles initiatives. Nos propos prennent appui sur notre jugement professionnel, fondé sur notre lecture des écrits consultés et sur nos expériences en pédagogie en tant qu'instigateurs d'initiatives et experts par expérience.

\section{Recommandations}

Mettre sur pied une initiative de formation en service social qui mise sur l'engagement actif des personnes ayant des incapacités exige la considération de plusieurs conditions par l'instigateur. D’abord, celui-ci doit prévoir différentes stratégies permettant de prévenir ou de réduire le déséquilibre de pouvoir pouvant émerger entre les personnes et les étudiants. Cette première condition paraît essentielle à une expression authentique et autonome des personnes impliquées (Towle et Godolphin, 2013a). De plus, la responsabilité de l'initiative devrait être partagée d'une manière qui convienne aux instigateurs tout comme aux personnes engagées, et ce, tant en ce qui concerne le développement des contenus que l'enseignement (team-teaching) et l'évaluation des apprentissages. 
Ensemble, ils doivent s'assurer que toutes les activités proposées sont directement liées aux objectifs visés et adaptées au niveau d'expérience des étudiants.

Or, lors des contacts avec les étudiants, des efforts soutenus devraient être déployés afin de s'assurer que la parole des personnes ait plus de poids et que leur implication soit perçue comme une opportunité. Dans ce but, l'instigateur doit adopter des façons de faire qui facilitent la prise de parole et qui mettent à profit la contribution unique de ces personnes, ce qui permet aussi, par le fait même, de modeler des savoir-faire et savoir-être essentiels en matière d'intervention.

Tel que le proposent Robinson et Webber (2013), les objectifs d'un tel engagement devraient toujours être clarifiés et discutés au préalable afin de s'assurer que les personnes recrutées comprennent bien les implications de leur engagement. Cela paraît aussi essentiel pour évaluer la mise en œuvre et l'atteinte de ces objectifs. Pour s'assurer d'une collaboration optimale avec les personnes ayant des incapacités impliquées, différentes mesures doivent être mises en place afin de répondre à leurs besoins généraux, en tant que personnes d'abord (p. ex. : tenir les activités à l'extérieur des heures de travail), et de leurs besoins particuliers, découlant de leurs limitations fonctionnelles (p. ex. : rembourser les frais de transport adapté) (Fox, 2011).

Le recrutement des personnes devrait aussi s'effectuer de manière à s'assurer qu'elles ont des contenus significatifs à transmettre et dont la portée sur l'apprentissage serait amoindrie s'ils étaient enseignés par des responsables de l'enseignement qui détiennent une compréhension théorique de leur réalité. Selon Towle et Godolphin (2013a), les contenus abordés devraient aussi correspondre à ce que ces personnes veulent que les étudiants apprennent, et ce, de la manière dont elles veulent l'enseigner. Respecter leur rythme et leurs façons de faire par une attitude et des gestes humanistes suscitant l'appropriation de leur pouvoir d'agir permet de modeler des compétences fort pertinentes pour l'intervention.

Pour assurer la mise en place de relations égalitaires entre ces personnes et les futurs travailleurs sociaux, l'instigateur d'une initiative de formation doit aussi favoriser la création d'un climat respectueux, dépourvu des stéréotypes et des préjugés entretenus de part et d'autre. Dans ce but, des activités visant la prise de conscience des fausses croyances de tout un chacun et l'identification de stratégies permettant de les dépasser devraient être proposées dès les premiers contacts.

Plusieurs auteurs rapportent que les initiatives isolées sont souvent insuffisantes et que la mise en place d'une stratégie systémique fondée sur la collaboration entre les institutions d'enseignement et la communauté est souvent nécessaire au succès et à la pérennité des projets de formation misant sur l'engagement actif des experts par expérience (Repper et Breeze, 2007; Towle et Godolphin, 2013b). Au minimum, elle requiert un engagement mutuel de la part des alliés mobilisés (Baldwin et Sadd, 2006).

Dans la mesure où l'on préconise l'engagement actif de personnes ayant des incapacités dans le but de favoriser l'intégration de valeurs et croyances propres au service social chez des apprenants, ces derniers doivent être incités à adopter une démarche réflexive concernant les dilemmes et enjeux se situant à l'intersection entre les valeurs et croyances de leur profession et celles résultant d'influences personnelles, culturelles et sociétales (Duffy et Hayes, 2012). Au-delà des valeurs de la profession, il importe aussi d'établir un cadre éthique et des procédures pour protéger les intérêts des personnes impliquées et les apprenants (Anghel et Ramon, 2009). 


\section{Conclusion}

L'engagement actif dans l'enseignement des personnes ayant des incapacités s'inscrit dans une volonté de susciter un réel changement de culture au sein des programmes de formation en service social. Cette stratégie pédagogique permet d'amener les futurs professionnels à percevoir les personnes ayant des incapacités bien au-delà de leur handicap en tant qu'individus à part entière et partenaires de l'intervention. De plus, le partage de savoirs et d'expertises paraît fort fécond pour tous, notamment grâce à une meilleure compréhension mutuelle de la réalité de l'autre. Multiplier les efforts en ce sens semble donc une approche fort pertinente pour amener les futurs travailleurs sociaux à percevoir les interactions comme des occasions de partenariats et pour accroître leur sensibilité aux besoins de toute personne, sans égard à sa condition.

MILOT, Élise

RUEST-PAQUETTE, Anne-Sophie

LABRECQUE, Robert

\section{RÉFÉRENCES}

Anghel, R., et S. Ramon (2009). " Service users and carers' involvement in social work education: Lessons from an English case study », European Journal of Social Work, vol. 12, n², p. 185-199.

Baldwin, M., et J. Sadd, J. (2006). «Allies with attitude! Service users, academics and social service agency staff learning how to share power in running social work education courses », Social Work Education, vol. 25, n 4, p. 348-359.

Beresford, P. (2000). "Service users' knowledges and social work theory: conflict or collaboration? 》, British Journal of Social Work, vol. 30, n 4, p. 489-503.

Bleakley, A., et J. Bligh (2008). «Students learning from patients: Let's get real in medical education » Advances in Health Sciences Education, vol. 13, nº 1, p. 89-107.

Bouquet, B. (2004). Éthique et travail social: une recherche du sens, Paris, Dunod.

Brodeur, N., et G. Berteau (2008). «La réflexion éthique: une dimension essentielle dans la pratique du service social », dans J.-P. Deslauriers et Y. Hurtubise, Introduction au travail social, Québec, Presses de l'Université Laval, p. 241-265.

Colby, I., et S.F. Dziegielewski (2010). Introduction To Social Work: The People's Profession, Chicago (IL), Lyceum Books.

Duffy, J., et D. Hayes (2012). «Social work students learn about social work values from service users and carers ", Ethics and Social Welfare, vol. 6, n 4, p. 368-385. 
Fox, J. (2008). "The importance of expertise by experience in mental health services " The International Journal of Leadership in Public Services, vol. 4, $n^{\circ} 4$, p. 39-43.

Fox, J. (2011). " "The view from inside": understanding service user involvement in health and social care education », Disability and Society, vol. 26, n², p. 169-177.

Jha, V., N.D. Quinton, H.L. Bekker et T.E. Roberts (2009). "Strategies and interventions for the involvement of real patients in medical education: A systematic review », Medical Education, vol. $43, n^{\circ} 1$, p. $10-20$.

McLaughlin. H. (2009). "What's in a name: "Client", "Patient”, “Customer”, "Consumer", "Expert by Experience“, "Service User"- What's next? », British Journal of Social Work, vol. 39, n6, p. 11011117.

Milot, É. (2016, décembre). «La participation active de personnes ayant des incapacités à la formation universitaire : que pouvons-nous apprendre d'une expérience menée à l'Université Laval? » Conférence offerte au Centre interdisciplinaire de recherche en réadaptation et intégration sociale, Québec.

Milot, É., A.-S. Ruest-Paquette, S. Letscher, G. Fortin et M.J. Dogba (soumis). « Active involvement of people with disabilities in education: A literature review », Journal of Human development, Disability and Social Change.

Repper, J., et J. Breeze (2007). « User and carer involvement in the training and education of health professionals: A review of the literature », International Journal of Nursing Studies, vol. 44, $\mathrm{n}^{\circ} 3$, p. 511-519.

Robinson, K., et M. Webber (2013). « Models and effectiveness of service user and carer involvement in social work education: a literature review », British Journal of Social Work, vol. 43, n5, p. 925944.

Spencer, J., W. Godolphin, N. Karpenko, et A. Towle (2011). Can patients be teachers? Involving patients and service users in healthcare professionals' education, Newcastle, The Health Foundation.

Towle, A., L. Bainbridge, W. Godolphin, A. Katz, C. Kline, B. Lown, I. Madularu, P. Solomon et J. Thistlethwaite (2010). "Active patient involvement in the education of health professionals ", Medical Education, vol. 44, $\mathrm{n}^{\circ} 1$, p. 64-74.

Towle, A., et W. Godolphin, W. (2013a). « Patients as educators: Interprofessional learning for patientcentred care », Medical Teacher, vol. 35, n³, p. 219-225.

Towle, A., et W. Godolphin (2013b). « Patient involvement in medical education » dans K. Walsh, The Oxford Textbook of Medical Education, Oxford, Oxford University Press, p. 311-322. 
Van de Sande, A., M.-A. Beauvolsk et G. Renault (2002). Le travail social: Théories et pratiques, Boucherville (QC), Gaëtan Morin Éditeur Itée. 